\title{
Lung ultrasound in the critically ill (LUCI): A translational discipline
}

\author{
Daniel Lichtenstein ${ }^{1}$, Manu L.N.G. Malbrain ${ }^{2,3}$ \\ ${ }^{1}$ Service de Réanimation Médicale, Hôpital Ambroise-Paré, Paris-West University, Paris, France \\ 2Intensive Care Unit and High Care Burn Unit, Ziekenhuis Netwerk Antwerpen, ZNA Stuivenberg, Antwerp, Belgium \\ ${ }^{3}$ Department of Intensive Care, University Hospital Brussels (UZB), Jette, Belgium and Faculty of Medicine, \\ Free University Brussels (VUB), Brussels, Belgium
}

\begin{abstract}
In the early days of ultrasound, it was not a translational discipline. The heart was claimed by cardiologists, with others, such as gynaecologists, urologists and vascular surgeons claiming their part while the rest was given to radiologists. Only recently, ultrasound transgressed and crossed the usual borders between the different disciplines, such as emergency and critical care medicine. The advent of portable machines in the early 1980s, allowed the critical care physician to perform bedside ultrasound, and the development of whole body critical care ultrasound (CCUS) was born. It may sound cynical that radiologists were the first to state that diagnostic sonography was truly the next stethoscope: poorly utilized by many but understood by few. Exactly the same radiologists then abandoned the use of ultrasound outside the radiology department, leaving a vast domain to other disciplines eager to welcome the modern stethoscope. In this review, we list the possibilities of lung ultrasound as a translational holistic discipline.
\end{abstract}

Anaesthesiology Intensive Therapy 2017, vol. 49, no 5, 430-436

Key words: lung, ultrasound; holistic; medicine, translational; CCUS; POCUS

Traditionally, ultrasound was not a translational discipline. The cardiologists claimed their part, as did gynaecologists and, later on, urologists and vascular surgeons. This was the case for decades. In the early 1980s, intensivists discovered ultrasound as a way to monitor heart function [1]. Initially, radiologists were concerned that untrained doctors were using ultrasound outside the radiology department. Dr Roy Filly, Professor Emeritus of Radiology, and chief of the department of diagnostic sonography at Stanford predicted in 1988 that ultrasound would likely become the new stethoscope: "As we look at the proliferation of ultrasound instruments in the hands of untrained physicians, we can only come to the unfortunate realisation that diagnostic sonography truly is the next stethoscope: poorly utilized by many but understood by few" [2]. The introduction of portable machines in the 1980s, allowed the intensivist to perform bedside ultrasound, and the development of whole body critical care ultrasound (CCUS). This movement likely inspired emergency physicians, who realized that the detection of blood collections could be of interest in trauma patients. At the same time, radiologists let go and no longer invested time, effort and knowledge in the use of ultrasound in the critically ill. Since the 2000s, critical care and emergency ultrasound has undergone a revolution. However, ICU and ER colleagues have shed new light on the most important piece of the puzzle, namely the lungs. CCUS of the lungs has made ultrasound the stethoscope of the $21^{\text {th }}$ century.

The consideration of lung ultrasound in the critically ill (LUCI) as a major point of interest makes ultrasound a true clinical tool $[3,4]$. It makes CCUS a holistic discipline. A discipline is holistic when each of its components, apparently unconnected, work together to make a whole. If lung ultrasound is not considered, the portable characteristics of the ultrasound machine, the Doppler button, and modern filters have nothing in common. When the lungs are added to the ultrasound examination, all of a sudden it can also be used in emergency situations, i.e. for demonstration of tension 
pneumothorax during cardiac arrest (obstructive shock). Therefore, a small portable machine is required, as there is no time (or need) to use a Doppler, while any modern filter is a hindrance (as explained in the SESAME-protocol) [3].

Although there are many clinical examples for the holistic use of $\mathrm{LUCl}$, we will illustrate it with a simple case. In a patient with acute dyspnoea due to hydrostatic pulmonary oedema, LUCI will show the B-profile, indicating left heart dysfunction [4]. Traditional transthoracic echocardiography will not visualize a "pulmonary oedema", but will show left ventricular dysfunction, which is not the actual problem but rather the cause.

The definition of holistic ultrasound points out that the use of $\mathrm{LUCl}$ can be extended to other settings, those less critical but seen in daily practice. This makes the basis of a translational vision of $\mathrm{LUCl}$. Here, we describe our experience from critical care to other, less critical disciplines.

\section{WHAT MAKES LUCI A TRANSLATIONAL TOOL?}

Translational medicine is a popular term often used nowadays. Although several disciplines may be interested in innovations, who would be interested in LUCI? In fact, many disciplines focus on the lungs, at one time or another. Briefly, all doctors using a stethoscope may be interested in LUCI. We will elaborate on this in the following sections of this article.

\section{THE INTENSIVIST'S PERSPECTIVE}

In a critically ill patient, the lungs are one of the body's most vital organs. Ironically, the lung was deemed as an obstacle to ultrasound use because of artefacts caused by air [5]. Nowadays, however, all doctors know that ultrasound helps us to diagnose various conditions that may explain the cause of acute respiratory failure. The BLUE-protocol has been designed for the rapid understanding of the etiology of respiratory failure, the FALLS-protocol for acute circulatory failure, and the SESAME-protocol for cardiac arrest.

We briefly describe the BLUE-protocol. A few areas of interest (two anterior, one posterior) are used, allowing one to analyze lung sliding, lung rockets, posterolateral alveolar and/or pleural syndrome (called PLAPS). By making associations between specific signs and the lung areas where they are present, the most common diagnoses can be made. This may be of interest when the clinical presentation is misleading. Hydrostatic pulmonary oedema is defined by the B-profile, which combines anterior lung sliding with anterior lung rockets. Some pneumonias are detected through the B'-profile, a specific sign, when lung rockets are seen with abolished lung sliding. The A-profile (anterior lung sliding without anterior lung rockets) associated with an area of deep venous thrombosis has a 99\% specificity for pulmonary embolism (in healthy lungs).
In the past many intensivists performed only echocardiography. However, recently, the BLUE protocol is also gaining its place. Some intensivists have also "tried" the FALLS-protocol in a more challenging field as this protocol handles acute circulatory failure, a setting with a limited gold standard, if any. To briefly describe the FALLS-protocol, the Fluid Administration Limited by Lung Sonography (FALLS) protocol rules out sequentially obstructive shock (pericardial tamponade, pneumothorax, pulmonary embolism and other causes of dilated right ventricle), and cardiogenic shock of left ventricular origin (absence of B-profile). After the exclusion of cardiogenic shock, fluid is administered, as it will be beneficial to all other sources of shock, hypovolaemic and distributive. This is a therapeutic test. Hypovolaemic shock should resolve itself under fluid therapy. In the FALLS-protocol, septic shock is defined by the transformation of A-lines to B-lines under fluid therapy. We briefly remind the reader that the FALLS-protocol allows one to redefine hydrostatic pulmonary oedema: when the circulating volume is under pressure, it creates a "pulmonary oedema", a term which is confusing, as this oedema begins in the interstitial space which does not take part in gas exchange (in French: "puisards septaux") [6]. Many physicians mistakenly understand "alveolar" oedema when they hear of "pulmonary" oedema. This is an opportunity for $\mathrm{LUCl}$, which can detect interstitial oedema through the lung rockets, at an early, infra-clinical step, one step ahead [7].

$\mathrm{LUCl}$ is holistic because it allows one to simplify cardiac evaluation. The Cercle des Echographistes d'Urgence et de Réanimation Francophones (CEURF) teaches an extremely simplified use of echocardiography, called simple emergency cardiac sonography (not echography, which is too specific, not ultrasound, which recalls examination beyond the heart alone). Here, the SLAM (Section for the Limitation of Acronyms in Medicine) did not see the need for an acronym. The combination LUCl/simple emergency cardiac sonography adds a new dimension in the assessment of the thorax (also called "the black box") at the bedside. We say "new" although all this has been described and published since 1994, even before. This ultrasound combination of lung and heart will be of major interest for those who have not yet attained the necessary skills (no beautiful windows) - those who have the skill and the windows, but who have to face the gray zone - and the most numerous on Earth, those who still do not have any echocardiographic machine.

The SESAME-protocol may be described in a few words. This approach to cardiac arrest is an opportunity for describing the seven requirements we ask a machine to provide in this setting but which works on less critical settings, namely: Requirement 1 - a very simple machine. Doppler, for instance, is not used here (and seldom in our emergency clinical work as the use of simple signs allows one to have the 
required clinical information). Moreover, filters are usually a hindrance when using $\mathrm{LUCl}$; Requirement 2 - a very small machine (for being used rapidly on-site), smaller than those normal-sized laptops which are too large. The ideal machine is compact (our machine, 32-cm width); Requirement 3 a fast start-up time (our machine, 7-seconds); Requirement 4 - a universal probe for a whole body but goal-directed approach; Requirement 5 - a perfect compromise for the image quality with the same probe and setting. This is required for the SESAME-protocol, but above all, it is the one we use daily for less-urgent tasks (subclavian catheterization etc); Requirement 6 - a flat keyboard, although not critical for cardiac arrest, it is absolutely essential in daily life for working with a clean machine; Requirement 7 - from the first and the fourth $\mathbf{L}$ a cost-effective machine just allows one to save more lives. Such a machine was already available from 1992 onwards, demonstrating that it was not necessary to wait for the laptop revolution. Indeed, 1992 technology (and even that of 1982) was perfect for the task at hand.

The SESAME-protocol is a really fast protocol since it rules out in a few seconds (shockable causes apart) the four main reversible causes of cardiac arrest, namely: tension pneumothorax (when the $A^{\prime}$-profile is identified); pulmonary embolism (using the BLUE-protocol when the lower femoral vein is clotted); abdominal bleeding; and pericardial tamponade. If none is positive, one has just to hope for a cardiac window, and mostly for the privilege of finding a reversible cause at this stage.

\section{KEY MESSAGES:}

- Lung and thoracic ultrasound in the critically ill, guided by different protocols (like BLUE, SESAME, FALLS,...) can help one to evaluate the etiology of cardiopulmonary failure, to manage pleural effusion, pneumothorax;

- It should be noted that vascular ultrasound can be used to detect deep venous thrombosis, to guide central venous or peripheral venous and arterial access, and to diagnose aortic syndromes.

\section{THE NEONATAL INTENSIVIST'S PERSPECTIVE}

Lung function in neonates is of critical concern. We have checked how far the signs observed and assessed in adults using suitable gold standards (i.e., CT) were visible in children [8]. The critically ill neonate shows exactly the same signs, no more, no less. With regard to lung ultrasound, the neonate's lungs is a miniature of adult lungs. Just imagine the change and how the physician will have critical information with minimal irradiation and no risk linked to transportation to radiology [9-11]. The neonate is one of the main targets of the LUCIFLR project (Lung Ultrasound in the Critically III Favoring Limitation of Radiation) [12].
KEY MESSAGE:

- The LUCIFLR project aims to limit, in the next three decades, the number of bedside radiographs by one-third, and mainly the number of urgent thoracic CTs by two-thirds.

\section{THE PAEDIATRICIAN'S PERSPECTIVE (NEONATES EXCLUDED)}

If the signs assessed in the adult were seen in the neonate, this means that the intermediate ages (infant, toddler, young child, adolescent...) will likely have the same benefits from $\operatorname{LUCl}[9,10]$.

The bottom line:

- The use of ultrasound in the paediatric population is virtually unlimited: from LUCI to the diagnosis of ureteral obstruction, intussusception, appendicitis and traumatic brain injury.

\section{THE ADULT CARDIOLOGIST'S PERSPECTIVE}

Cardiologists use sophisticated approaches for assessing the left heart function. Although they have used ultrasound probes for decades and carefully studied the heart, this only concerned the heart. Thus, if cardiologists had tilted the probe a little bit more towards the left, they would also have visualized a small part of the lung, a vital organ so close to the heart. Imagine the potential of a discipline which can associate, or dissociate, cardiologic from lung findings (the presence or absence of the B-profile in patients with or without visible left dysfunction, or just in the grey zone, or just without cardiac window). Fortunately, the cardiologic community is beginning to take interest in $\operatorname{LUCl}[13,14]$.

What is valuable during the management of shock can be used in the same way in more stable conditions, such as early diagnosis of cardiogenic (hydrostatic) pulmonary oedema, at a sub-clinical level [7]. Examining the lungs in addition to the heart should take less than one minute. In the BLUE-protocol, the time devoted in search of a B-profile is roughly 30 seconds.

\section{KEY MESSAGE:}

- We are convinced that in the future, patients will have a combined heart/lung ultrasound examination, allowing simultaneous visualization of the cause and the consequence of a disease.

\section{THE PAEDIATRIC CARDIOLOGIST'S PERSPECTIVE}

As cardiology is usually linked to the adult population, let us devote one sentence to those who deal with congenital heart diseases in children, both a small and huge world. LUCI should have a major place in this population as well.

\section{THE ANAESTHESIOLOGIST'S PERSPECTIVE}

As some anaesthesiologists are also intensivists, they may have already read the section devoted to the intensiv- 
ist. Outside the critical care setting, there is a huge field of application. With the compact ultrasound machines that were already available since 1982, anaesthetists could have been able to use CCUS to insert central venous catheters with nearly zero fault, to control fluid and bleeding losses during surgery, just to name a few examples. Transesophageal echocardiography (TEE) is very useful where there is no access to the thorax. Lung ultrasound can be a good alternative, provided a small space is devoted at the level of the upper lungs. This is enough for performing a FALLSprotocol, but also in conditions where the thorax has been opened (which is a limitation of TEE, since the pleural variations are altered).

\section{KEY MESSAGES:}

- Ultrasound allows for the recognition of high-risk patients before surgery: by scanning the lung, the anaesthesiologist will better recognize patients with clinically occult left heart dysfunction.

- Ultrasound allows for the assessment of fluid status during general surgery in order to replace correctly the losses from using indirect tools.

- New habits in the OR should include scanning of the lungs during abdominal surgery. We have written that since 1992: "If ultrasound succeeds penetrating the prestigious operating room, it can initiate a small revolution" [4]. Fortunately, while ultrasound has gained its place in the $\mathrm{OR}$, it took a long time.

\section{THE THORACIC SURGEON'S PERSPECTIVE}

Before surgery, it is important to detect adhesions (by abolished lung sliding). During surgery, a lung exclusion should be checked with ultrasound. After a pulmonectomy, the initial pattern is the $A^{\prime}$-profile. This is logical since, in fact, we face, stricto sensu, a "pneumo-thorax". The cavity is little by little filled with fluid/blood. In cases where a "swirl sign" is visible and the physician interprets this sign as a classic pneumothorax, needle insertion would not have dramatic consequences. After a pulmonectomy, the intra-thoracic pressures must be balanced between the residual air and the contralateral lung. The gold standard (mediastinal location on bedside radiography) may be replaced by ultrasound.

\section{KEYMESSAGE:}

- Lung ultrasound can be the new stethoscope for the thoracic surgeon in the postoperative follow-up after a pulmonectomy.

\section{THE NEPHROLOGIST'S PERSPECTIVE}

Nephrologists are interested in the volume status of their patients (especially if they are oliguric or anuric and on chronic dialysis). Some may use the stethoscope in search for crepitations to predict the need for dialysis, although this may be a sign of alveolar oedema. Others may use changes in dry weight and body weight or bio-electrical impedance analysis. Using LUCI they will have a subtler vision of lung function, detecting interstitial oedema at an early stage. Likewise, lung rockets indicate wet lungs, and A-lines dry lungs [15]. Ultrasound also allows one to measure the renal resistive index which has been shown to be correlated with intra-abdominal pressure [16].

\section{KEY MESSAGE:}

- In patients with cardio-renal dilemma or cardio-abdominal renal syndrome (CARS) $\mathrm{LUCl}$, combined with an abdominal ultrasound, can help to identify patients with congestive heart failure that may be at risk for worsening renal failure [17].

\section{THE EMERGENCY CARE PHYSICIAN'S PERSPECTIVE}

Developing ultrasound use in the emergency room (with a fast protocol) is of special interest, both in critically ill unstable patients but also in non-critically ill patients presenting with lung problems.

It seems that emergency physicians only took interest in ultrasound when ultrasound machines at last became "small" enough for them to be able to be used outside the radiology department. This is one misconception in the history of acute medicine, since machines from 1992, and even 1982, were not only as small or smaller than modern laptops, but even technically better for CCUS. It should be noted that the concept of using modern laptop machines was mostly guided by radiologists or cardiologists, unaware of the potentials of LUCI. Therefore, they focused on minimizing the artefacts caused by the lungs, as well as the subtle dynamic signs of LUCl. This explains a 30-year step backward, a unique phenomenon in medicine. In the first years of the laptop revolution, emergency physicians had to deal with large machines, poor image quality, long startup times, complex keyboards devoted to specialists, with the three normal probes but not a universal one, harmonic filters, and, last but not least, increased costs. In retrospect, we paved the way much earlier, having used, since 1985, bedside technology dating from 1982 which laid the basis of the first publication on the BLUE-protocol [18].

The bottom line:

- Ultrasound in the emergency room includes not only LUCI but all critical care, extended abdominal, testicular, early obstetric, musculoskeletal, and ocular ultrasonography [19].

- Typically, a single examination is necessary for diagnosis and disposition.

- Frequently leads to a decision to discharge the patient from hospital. 


\section{PRE-HOSPITAL MEDICINE}

In 1994, the company Dymax (Pittsburgh) produced a $3.5 \mathrm{~kg}$ portable TM-18 machine allowing us to use ultrasound in an African mission on a helicopter. With this device, the first pre-hospital ultrasound diagnosis of pneumothorax was made in the Saharan desert [20]. Of course, if ultrasound was possible in the sky, it could be of even more value in a simple ambulance. We are glad to have given the idea of pre-hospital ultrasound to the community [20].

Subsequently, we have been using a portable machine (1850 $\mathrm{g}$ with its own unique probe, kept in a box of $15 \times$ $12 \times 12 \mathrm{~cm}$ ) during airborne missions since 1998 . Although this unit came from the veterinary domain, it has allowed us to save human lives.

\section{KEY MESSAGES:}

- The traditional dilemma of "scoop and run" versus "play and stay" is solved when visual medicine is used on-site.

- We recommend that young ER doctors start their CCUS training with the lungs before trying to perform expert echocardiography in an emergency setting, as holistic ultrasound allows one to use the lung to answer cardiac questions (as explained above).

- Investing in the heart without examining the lung is not holistic ultrasound.

\section{THE FLYING DOCTOR'S PERSPECTIVE}

Ultrasound can be helpful during medical retrievals. The ULTIMAT-protocol (ultrasound limited test initiating medical airborne transportation) is an approach that we use routinely when transporting critically ill patients. The focus is on occult but possible lethal conditions: mainly pneumothorax, floating DVT, pericardial effusions close to the tamponade, amongst others.

\section{KEY MESSAGES:}

- Diagnosing a pneumothorax clinically during flight is very difficult.

- In contrast, the ultrasound approach gives one a proper visualization, which does not suffer from background noise making auscultation or percussion of the chest difficult to interpret.

- Those settings where space is really a hindrance are a good opportunity for using hand-held machines.

\section{THE GYNECO-OBSTETRICIAN'S PERSPECTIVE}

These doctors are often faced with severe conditions such as (pre)eclampsia, toxaemia of pregnancy, amniotic pulmonary embolism, critical hypovolaemia from bleeding (HELLP syndrome, namely haemolysis elevated liver enzymes and low platelets). In case of ovarian hyperstimulation syndrome, ascites can be detected and drained [21].
The simple question regarding pulmonary oedema is solved in 30 seconds using the BLUE-protocol.

\section{KEY MESSAGES:}

- In pulmonary embolism, the BLUE-protocol allows a diagnosis to be made in most cases.

- In cases of massive blood loss, the FALLS-protocol can guide one between the risks of hypovolaemia and fluid overload (therapeutic dilemmas).

\section{INTERNAL MEDICINE PHYSICIAN'S PERSPECTIVE}

All internal medicine physicians have, without exception, a stethoscope. However, some diseases have no known findings on physical examination. Except for the presence of fine crepitations," crisdes petits oiseaux" (the chirping of little birds) or squeaks [22], the stethoscope is of no use when assessing interstitial disease (extrinsic allergic alveolitis, lung fibrosis), whereas ultrasound can document the presence or absence of lung rockets rapidly.

\section{KEY MESSAGE:}

- The time is right for internists adopt the handheld ultrasound device, without the intent of replacing the traditional stethoscope.

\section{THE PULMONOLOGIST'S PERSPECTIVE}

The word "pulmonology" is derived "pulmon", i.e., lung and it is likely that pulmonologists will use ultrasound one day. It is true that they are accustomed to high-resolution CT images, which outline subtle details of chronic interstitial diseases better than ultrasound.

Pulmonologists could, however, take some responsibility for decreasing the radiation doses [23].

\section{KEY MESSAGES:}

- By definition, pneumologists or lung specialists should be the ambassadors for LUCI.

- The diagnosis and management of pleural syndromes, biopsy of subpleural lesions are possible applications.

- Recently, they have begun to appreciate the potential of ultrasound.

\section{THE FAMILY DOCTOR'S PERSPECTIVE}

In deep winter, family doctors often have to deal with a febrile child carried in their mother's arms. With LUCI, however, she will not need to leave with a prescription for a radiograph and will have a diagnosis and treatment on-site.

\section{KEY MESSAGES:}

- In patients presenting with vague respiratory symptoms, $\mathrm{LUCl}$ can detect chronic interstitial syndromes.

- In other situations, time can be saved (differential diagnosis of chest pain, etc). 


\section{THE TRAUMA SURGEON'S PERSPECTIVE}

In some areas, the doctor managing a trauma patient is the surgeon. In trauma, physical examination is usually aspecific.

\section{KEYMESSAGE:}

- LUCI will provide trauma surgeons with an immediate and correct diagnosis (pneumothorax, lung contusion, haemothorax...).

\section{THE GERIATRICIAN'S PERSPECTIVE}

This is a young discipline [24]. Imagine how many times they rely on physical signs and auscultation. $\mathrm{LUCl}$ can be used without adaptation in order to detect disorders that are at best treated at an early stage.

\section{KEYMESSAGE:}

- LUCI has a real future in this growing population

\section{ULTRASOUND IN THE (DEVELOPING) WORLD}

Here is probably the largest number of patients. A simple radiography is a luxury. As part of the developed world, we must embrace the people living in these deprived and remote areas. We present the SHUFLES program (Simple Holistic Ultrasound For Low-Economy Settings) as a holistic and translational vision. The SHUFLES program uses the same simple machine, one single probe and no Doppler or sophisticated modalities, but allows for similar diagnostic possibilities as a mini-bedside CT.

\section{KEY MESSAGES:}

- This is one application of holistic ultrasound: no adaptation is necessary between sophisticated Western ICUs and these remote areas.

- The signs are the same. A consolidation is a consolidation. Whole body ultrasound could make a major difference in the therapeutic decisions taken.

\section{OTHER "ISOLATED" DOCTORS' PERSPECTIVES}

Some doctors work on cruise ships. Here potential patients usually can afford extra care. These doctors need to master physical examination, as this is often the only thing they can rely on. As only few have a real "small hospital" available on board with X-rays, a lab, the possibility of urgent coronary care, why not add an ultrasound machine? Some doctors work in the jungle, or in settings with really poor facilities. They would enjoy the use of small, light equipment.

\section{MISCELLANEOUS}

People performing acupuncture are sometimes nervous as, on rare occasions, they create a pneumothorax. However, asking for a traditional imaging after each session would be a disaster. Indeed, requesting a radiograph would generate irradiation, cost, loss of time with only a minor benefit if the pneumothorax is initially not detectable radiologically. $A$ rapid ultrasound view would allow one to select in a few seconds those who need to be transferred to hospital.

Whilst performing functional evaluations, doctors will better understand lung physiology, as lung sliding is a dynamic sign that can only be demonstrated by ultrasound.

During palliative care, diagnostic escalation is not the first option, but ultrasound is precisely the opposite. It will on occasion highlight reversible causes of severe discomfort.

Many respiratory therapists are interested in $\mathrm{LUCl}$, as it is perfectly suitable for bedside real-time assessment. Sonographers may also play a role, as they are familiar with the technique involved.

\section{MEDICAL STUDENT'S PERSPECTIVE}

The best way to make $\mathrm{LUCl}$ a reality would be to incorporate it into medical education. Not whole-body ultrasound, as this would result in making medical studies longer with an insignificant benefit (what percentage of them would ever benefit from the mastery of a biliary tract ultrasound?). However, it would be sensible to start with a lung ultrasound, not only as it is simple but because all future doctors dealing with the lung will benefit from it.

\section{ALL DOCTORS}

The LUCIFLR project concerns all those who require chest X-rays or CTs. Imagine that the access to CT may become restrained in the future in order to limit radiation [12]. As this is not a hypothetical consideration, one better be prepared (especially in specific settings, if for instance if the only question is the presence or absence of pneumothorax).

\section{“LAST BUT NOT LEAST" LUCIA, LUNG ULTRASOUND IN CRITICALLY ILL ANIMALS. THE VETERINARIAN'S PERSPECTIVE}

We recall that the pocket machine we used since 1998 was, ironically, from the veterinarian world. As LUCI works perfectly in humans, without any known side effects, we could also apply this discipline to our pet animals. Every animal possessing lungs can benefit from $\mathrm{LUCl}$. We extrapolate exactly the same signs. As with critically ill patients, animals do not speak, thus, one may imagine the benefits.

\section{CONCLUSIONS}

Each time the word "lung" is uttered i.e. many times a day in the above-listed disciplines, ultrasound has a place at the bedside in order to solve clinical questions. This results in rapid diagnosis, cost savings, less suffering, and the birth of a new form of medicine based on visual bedside observations. Within practiced hands, ultrasound can replace CT (and of course $X$-rays), namely the LUCI-FLR project. As the official imaging 
specialists, the radiologists have now an important role to play as they need to accept the importance of lung ultrasound. This would add another specialty to the long list. Some clinicians are still reluctant to perform lung ultrasound as the air in the lungs is perceived as a major limitation while intensivists and emergency physicians use these artefacts to make a proper diagnosis [25]. Therefore, more effort is needed to facilitate the widespread adoption by the medical community of lung ultrasound.

\section{ACKNOWLEDGEMENTS}

1. This article is endorsed by the International Fluid Academy (IFA). The mission statement of the IFA is to foster education, promote research on fluid management and haemodynamic monitoring, and thereby improve the survival of the critically ill by bringing together physicians, nurses, and others from throughout the world and from a variety of clinical disciplines. The IFA is integrated within the not-for-profit charitable organization iMERiT, International Medical Education and Research Initiative, under Belgian law. The IFA website (http://www.fluidacademy. org ) is now an official SMACC-affiliated site (Social Media and (Critical Care) and its content is based on the philosophy of FOAM (Free Open Access Medical education \#FOAMed). The site recently received the HONcode quality label for medical education (https://www.healthonnet. org/HONcode/Conduct.html?HONConduct519739).

2. Conflicts of interest: Manu .N.G. LMalbrain is founding President and current Treasurer of WSACS (The Abdominal Compartment Society). He is also member of the medical advisory Board of Pulsion Medical Systems (part of Maquet Getinge group) and consults for ConvaTec, Acelity, Spiegelberg and Holtech Medical. Manu Malbrain is co-founder of the International Fluid Academy (IFA). Daniel Lichtenstein has no possible conflicts of interest in relation to the content of this review article.

\section{References:}

1. Jardin F, Farcot JC, Boisante L, et al. Influence of positive end-expiratory pressure on left ventricular performance. N Engl J Med. 1981; 304(7): 387392, doi: 10.1056/NEJM198102123040703, indexed in Pubmed: 7005679.

2. Filly RA. Ultrasound:the stethoscope of the future, alas. Radiology. 1988; 167(2): 400, doi: 10.1148/radiology.167.2.3282260, indexed in Pubmed: 3282260.

3. Lichtenstein D, Malbrain ML. Critical care ultrasound in cardiac arrest. Technological requirements for performing the SESAME-protocol--a holistic approach. Anaesthesiol Intensive Ther. 2015; 47(5): 471-481, doi: 10.5603/AIT.a2015.0072, indexed in Pubmed: 26578398.

4. Lichtenstein $D$, van Hooland $S$, Elbers $P$, et al. Ten good reasons to practice ultrasound in critical care. Anaesthesiol Intensive Ther. 2014; 46(5): 323-335, doi: 10.5603/AIT.2014.0056, indexed in Pubmed: 25432552.

5. Fuhlbrigge AL, Choi AMK. Diagnostic procedures in respiratory disease. In: Kasper DL, Fauci AS, Longo DL, Hauser SL, Loscalzo J. ed. Harrison's Principles of internal medicine. McGraw-Hill, New York 1663.

6. Rémy-Jardin $M$, Rémy J. CEdème interstitiel. In: Rémy-Jardin $M$, Rémy J. ed. Imagerie nouvelle de la pathologie thoracique quotidienne. Springer-Verlag, Paris 1995: 137-143.

7. Gargani L, Lionetti V, Di Cristofano C, et al. Early detection of acute lung injury uncoupled to hypoxemia in pigs using ultrasound lung comets. Crit Care Med. 2007; 35(12): 2769-2774, doi: 10.1097/01. CCM.0000287525.03140.3F, indexed in Pubmed: 17828031.
8. Lichtenstein DA, Mauriat P. Lung Ultrasound in the Critically III Neonate. Curr Pediatr Rev. 2012; 8(3): 217-223, doi: 10.2174/157339612802139389, indexed in Pubmed: 23255876.

9. Copetti R, Cattarossi L. The 'double lung point': an ultrasound sign diagnostic of transient tachypnea of the newborn. Neonatology. 2007; 91(3): 203-209, doi: 10.1159/000097454, indexed in Pubmed: 17377407.

10. Tsung JW, Kessler DO, Shah VP. Prospective application of clinician-performed lung ultrasonography during the $2009 \mathrm{H} 1 \mathrm{~N} 1$ influenza A pandemic: distinguishing viral from bacterial pneumonia. Crit Ultrasound J. 2012; 4(1): 16, doi: 10.1186/2036-7902-4-16, indexed in Pubmed: 22862998.

11. Caiulo VA, Gargani L, Caiulo S, et al. Lung ultrasound in bronchiolitis: comparison with chest X-ray. Eur J Pediatr. 2011; 170(11): 1427-1433, doi: 10.1007/s00431-011-1461-2, indexed in Pubmed: 21468639.

12. Brenner D, Elliston C, Hall E, et al. Estimated risks of radiation-induced fatal cancer from pediatric CT. AJR Am J Roentgenol. 2001; 176(2): 289-296, doi: 10.2214/ajr.176.2.1760289, indexed in Pubmed: 11159059.

13. Vermeiren GLJ, Malbrain ML, Walpot JM. Cardiac Ultrasonography in the critical care setting: a practical approach to asses cardiac function and preload for the "non-cardiologist". Anaesthesiol Intensive Ther. 2015; 47 Spec No: s89-104, doi: 10.5603/AIT.a2015.0074, indexed in Pubmed: 26588484.

14. Poelaert J. Assessment of loading conditions with cardiac ultrasound. A comprehensive review. Anaesthesiol Intensive Ther. 2015; 47(5): 464-470, doi: 10.5603/AIT.a2015.0068, indexed in Pubmed: 26505579.

15. Noble VE, Murray AF, Capp R, et al. Ultrasound assessment for extravascular lung water in patients undergoing hemodialysis. Time course for resolution. Chest. 2009; 135(6): 1433-1439, doi: 10.1378/chest.08-1811, indexed in Pubmed: 19188552.

16. Kirkpatrick AW, Colistro R, Laupland KB, et al. Renal arterial resistive index response to intraabdominal hypertension in a porcine model. Crit Care Med. 2007; 35(1): 207-213, doi: 10.1097/01.CCM.0000249824.48222.B7, indexed in Pubmed: 17080005.

17. Verbrugge $F H$, Dupont $M$, Steels $P$, et al. Abdominal contributions to cardiorenal dysfunction in congestive heart failure. J Am Coll Cardiol. 2013; 62(6): 485-495, doi: 10.1016/j.jacc.2013.04.070, indexed in Pubmed: 23747781.

18. Lichtenstein DA, Mezière GA. Relevance of lung ultrasound in the diagnosis of acute respiratory failure: the BLUE protocol. Chest. 2008; 134(1): 117-125, doi: 10.1378/chest.07-2800, indexed in Pubmed: 18403664.

19. Whitson MR, Mayo PH. Ultrasonography in the emergency department. Crit Care. 2016; 20(1): 227, doi: 10.1186/s13054-016-1399-x, indexed in Pubmed: 27523885.

20. Lichtenstein D, Courret JP. Feasibility of ultrasound in the helicopter. Intensive Care Med. 1998; 24(10): 1119, indexed in Pubmed: 9840254.

21. Kumar P, Sait SF, Sharma A, et al. Ovarian hyperstimulation syndrome. J Hum Reprod Sci. 2011; 4(2): 70-75, doi: 10.4103/0974-1208.86080, indexed in Pubmed: 22065820.

22. Earis JE, Marsh K, Pearson MG, et al. The inspiratory "squawk" in extrinsic allergic alveolitis and other pulmonary fibroses. Thorax. 1982; 37(12): 923-926, indexed in Pubmed: 7170682.

23. Lichtenstein D. The LUCI-FLR Project: lung ultrasound in the critically III - a bedside alternative to irradiating techniques, radiographs and CT. Lung ultrasound in the critically III. 2016: 217-225, doi: 10.1007/9783-319-15371-1_29.

24. Morley JE. Geriatricians: The Super Specialists. J Am Geriatr Soc. 2017; 65(4): 866-868, doi: 10.1111/jgs.14702, indexed in Pubmed: 28177517.

25. van der WerfTS, Zijlstra JG. Ultrasound of the lung: just imagine. Intensive Care Med. 2004; 30(2): 183-184, doi: 10.1007/s00134-003-2083-6, indexed in Pubmed: 14685657.

\section{Corresponding author:}

Manu L.N.G. Malbrain

Department of Intensive Care Medicine

and High Care Burn Unit

Ziekenhuis Netwerk Antwerpen

ZNA Stuivenberg Hospital, Antwerp, Belgium

e-mail:manu.malbrain@uzbrussel.be

Received: 16.10 .2017

Accepted: 11.11.2017 